\title{
Development and Validation of a Stability-Indicating RP-UPLC Method for the Quantitative Analysis of antiparkinson drug and its related impurities
}

\author{
N.Balaji ${ }^{1}$, V.R.Sivaraman ${ }^{1}$, Dr.P. Neeraja ${ }^{2}$ \\ ${ }^{I}$ (Department of Analytical chemistry, Dravidian University, Kuppam, India) \\ ${ }_{2}^{2}$ (Department of chemistry, Adhiyamaan engineering college, Anna University, Hosur, India)
}

\begin{abstract}
High efficiency, cost effectiveness and short run time are the basic requirements of high-speed chromatographic separations. To fulfill these requirements, an advanced separation technique, ultraperformance liquid chromatography (UPLC), has shown promising developments. A rapid, specific, sensitive, and precise reverse-phase UPLC method was developed for the determination of antiparkinson drug and its impurities. In this work, a new gradient reverse phase chromatographic method using UPLC for the determination was done for active pharmaceutical ingredient in the presence of degradation products, and its process related impurities.The chromatographic separation was achieved on a Waters Acquity BEH C18 column $(50 \mathrm{~mm}, i . d ., 2.1 \mathrm{~mm}, 1.7 \mu \mathrm{m})$ within a short runtime of 4 minutes using a mobile phase of $5 \mathrm{mM}$ potassium phosphate and acetonitrile, at a flow rate of $0.5 \mathrm{~mL} / \mathrm{min}$ at an ambient temperature. Forced degradation studies were also performed for antiparkinson bulk drug samples to demonstrate the stabilityindicating power of the UPLC method. Linearity range was LOQ-200\% with respect to $100 \mu \mathrm{g} \mathrm{mL} L^{-1}$ of active pharmaceutical ingredient for related impurities; Linearity range was $40-160 \%$ with respect to $10 \mu \mathrm{g} \mathrm{mL} \mathrm{L}^{-1}$ of active pharmaceutical ingredient for assay calculated by 5 determinations. Comparison of system performance with conventional high-performance liquid chromatography was made with respect to analysis time, efficiency, and sensitivity. The method is validated according to the ICH guidelines and is applied successfully for the quantitative determination of antiparkinson drug and its related impurities.
\end{abstract}

Keywords: Ultra performance liquid chromatography (UPLC), Antiparkinson drugs, Validation and quantification

\section{Introduction}

A new category of separation technique, ultra-performance liquid chromatography (UPLC), has proven to be one of the most promising developments in the area of fast chromatographic separations with its unique characteristics of high chromatographic resolution, speed, and sensitivity analysis [1-4]. It utilizes sub-2 micron particles for the stationary phase. These particles operate at elevated mobile phase linear velocities to affect dramatic increase in efficiency [5]. In the present work, this technology has been applied to the method development, validation, and determination of rasagiline mesylate and quantification of its related impurities.

Rasagiline mesylate was chosen from list of antiparkinson drugs. Rasagiline mesylate (API) is an irreversible inhibitor of monoamine oxidase [MAO][6] used as amonotherapy in early Parkinson's disease or as an adjunct therapy in more advanced cases[7]. It is selective for MAO type B over type A by a factor of fourteen [8].Human cells contain two forms of monoamine oxidase, MAO-A and MAO-B. Both are found in the brain, but MAO-B is far more prevalent and is responsible for the breakdown of dopamine after its release into the synapse. Parkinson's disease is characterized by the death of cells that use dopamine to transmit their signals, these results in a decrease in synaptic signal strength and concomitant symptomology. By inhibiting the breakdown of dopamine in the synapse, rasagiline permits the signaling neurons to reabsorb. Rasagiline is broken down via Cytochrome P450 1A2 [CYP1A2] [9], part of the cytochrome P450 metabolic path in the liver. It is probably contraindicated in patients with hepatic insufficiency and its use should be monitored carefully in patients taking other drugs that alter the normal effectiveness of this metabolic path.

Ultra performance liquid chromatography (UPLC) has been considered as a novel development in liquid chromatography. It is specially designed to withstand higher system pressures during chromatographic analysis so that it enables significant decrease in separation time and solvent consumption. The UPLC columns packed with $1.7 \mu \mathrm{m}$ sized particles provides not only increased efficiency but also the ability to work at increased linear velocity without loss of efficiency, providing both resolution and speed. Using advantages of UPLC, a number of applications in different fields including pharmacy [10], clinical analysis, pesticide analysis [11] and tetracyclines in human urine [12] have been reported. For structural elucidation accurate mass data gives the reliable information which can confirm the molecular formula of a compound [13]. QToF-MS provides exact 
mass measurements for both precursor and fragment ions to yield high confidence in structural elucidation [14].The coupling of UPLC with QToF-MS/MS extends high chromatographic resolution with exact mass measurement for both MS and MS/MS, and then allows significant advantages of speed, selectivity, sensitivity and accuracy for rapid screening of known as well as unknown compounds.

In this work, the HPLC method for rasagiline mesylate was transferred to UPLC. A comparison was made between HPLC and UPLC efficiency on the basis of resolution and sensitivity. The developed stabilityindicating reversed-phase (RP) UPLC method was then validated as per ICH guidelines [15], along with the application of the developed method in the determination of rasagiline mesylate and quantification of its related impurities. However, there are no reports available on stability indicating RP-UPLC analytical method for determination of rasagiline mesylate and quantification of its related impurities with short run time. It was, therefore, necessary to develop a new rapid stability-indicating method for the quantitative estimation of rasagiline mesylate and its related impurities. This work clearly explains about how the UPLC can reduce analysis times without compromising the resolution.During the literature survey of the API, different analytical methods were reported for determination of API by HPLC [16-18], UV Spectrophotometry [19-20] and mass spectrometry [21].

\subsection{Chemicals}

\section{Experimental Data}

Rasagiline mesylate and its impurities were provided by Zhangshu Pharma limited (China). All HPLC grade solvents were obtained from Fisher Scientific (Mumbai, India). Water $(18.2 \mathrm{~m} \Omega \mathrm{cm}$ ) was obtained using a Milli-Q system (Millipore, Bangalore, India). Monobasic Potassium phosphate $\left(\mathrm{KH}_{2} \mathrm{PO}_{4}\right)$ and Sodium hydroxide $(\mathrm{NaOH})$ were obtained from Fisher Scientific (Mumbai, India). All solutions were filtered through $0.22 \mu \mathrm{m}$ nylon filters manufactured by Millipore Pvt. Ltd (Bangalore, India).The structures of API and related compounds listed in Fig. 1.

\section{Fig.1 Structures}

\subsection{Equipment} HPLC

The HPLC system used for initial chromatographic development was Waters Alliance separation module with a 2996 PDA detector (Waters, Milford Massachusetts). 
UPLC

UPLC was performed using a Waters Acquity system equipped with binary solvent delivery pump, an auto sampler and PDA detector (Waters).

Cintex digital water bath was used for acid and base hydrolysis studies. Photostability studies were carried out in a Sanyo Photostability chamber (Leicestershire, UK). Thermal stability studies were performed in a dry air oven from Thermo (Mumbai, India). Intermediate precision study was performed on different Waters Acquity UPLC ${ }^{\mathrm{TM}}$ system (Milford, USA) consists of a binary solvent manager, a sample manager and a tunable ultraviolet (TUV) detector. All UPLC systems were equipped with a column compartment with temperature control and an on-line degasser. Data acquisition, analysis, and reporting were performed, Empower2 (Waters) chromatography software.

\subsection{Chromatographic conditions}

HPLC: Chromatographic separation was achieved in gradient elution mode. A Sunfire C18, 250 x 4.6 $\mathrm{mm}, 5 \mu \mathrm{m}$ column was used for gradient separation (Waters, NY). A mobile phase consisting of A: $5 \mathrm{mM}$ potassium phosphate with $\mathrm{pH} 7.0$ using 10\% sodium hydroxide (Buffer) solution and B: acetonitrile for timed gradient program $\mathrm{T}(\mathrm{min}) / \% \mathrm{~B}: 0 / 5,25 / 30,35 / 50,45 / 50,45.1 / 5,50 / 5$, with the flow rate of $1 \mathrm{~mL} / \mathrm{min}$ was employed. The injection volume was $10 \mu \mathrm{L}$ while detector was set at $210 \mathrm{~nm}$. The column was maintained at $25^{\circ} \mathrm{C}$.

UPLC: The mobile phase used was a mixture of ACN and $5 \mathrm{mM}$ potassium phosphate employing gradient elution. The UPLC gradient was set as: T/\%B: 0/5, 2.08/30, 2.92/50, 3.75/50, 3.77/5, 4.17/5. The detector was set at a sampling rate of 20 points $\mathrm{s}^{-1}$ and filter time constant of $0.2 \mathrm{~s}$. System control, data collection and data processing were accomplished using Waters Empower chromatography data software. The analytical column used was $50 \mathrm{~mm} \times 2.1 \mathrm{~mm}, 1.7 \mu \mathrm{m}$ Waters Acquity UPLC BEH C-18 column (Milford, USA). The optimized conditions were as follows: injection volume: $2.0 \mu \mathrm{L}$, flow rate: $0.5 \mathrm{~mL} \mathrm{~min}{ }^{-1}$ at a column temperature of $25^{\circ} \mathrm{C}$, sample cooler temperature of $25^{\circ} \mathrm{C}$ and detection wavelength: $210 \mathrm{~nm}$. Under these conditions the system back pressure was about 9600 psi.

\subsection{Preparation of solutions}

\subsubsection{Standard stock solutions}

The individual standard stock solutions of API and its impurities were prepared by dissolving corresponding standard compounds in diluent (Diluent was 50:50 of ACN and Buffer) to obtain a concentration of 100,150 and $150 \mu \mathrm{g} \mathrm{mL}^{-1}$, respectively. $1 \mathrm{~mL}$ of all standard stock solution was further diluted to $100 \mathrm{~mL}$ with diluent and used as the standard to quantify the related impurities.

\subsubsection{System suitability solution}

System suitability parameters were measured in order to verify the system performance. The system suitability solution was prepared to obtain $1.0,1.5$ and $1.5 \mu \mathrm{g} \mathrm{mL}{ }^{-1}$ concentrations of API, Imp-A and Imp-B respectively using standard stock solutions. Six replicate injections of the system suitability solution were performed to measure the important characteristics including peak resolution, tailing factor and theoretical plates.

\subsubsection{Sample preparation}

Transferred $5 \mathrm{mg}$ of API into a $50 \mathrm{~mL}$ volumetric flask, added $35 \mathrm{~mL}$ of the diluent and sonicated for 5 min. Keep the solution aside to attain room temperature, then make up to the volume with diluent and filtered through $0.22 \mu \mathrm{m}$ nylon filter. This solution was employed as sample solution for quantification of its related impurities $\left(100 \mu \mathrm{g} \mathrm{mL} L^{-1}\right) .5 \mathrm{~mL}$ of this solution was diluted to $50 \mathrm{~mL}$ with diluent and filtered, used as the assay sample solution $\left(10 \mu \mathrm{g} \mathrm{mL}^{-1}\right)$.

\subsection{LC Method development}

\section{Results And Discussion}

The HPLC method carried out in this study aimed at developing chromatographic system capable of eluting and resolving Rasagiline mesylate from its process related impurities and degradation products that comply with the general requirements for system suitability. Initial trials were done on Inertsil ODS 2 C18 column $(150 \mathrm{~mm} \times 4.6 \mathrm{~mm}$ i.d., particle size $5 \mu \mathrm{m})$ with mobile phase, $0.1 \%$ Formic acid: Acetonitrile with the gradient as (time (min)/\% solution B): $0 / 25,5 / 25,45 / 30,50 / 30,51 / 75,55 / 75$ at flow rate $1.0 \mathrm{~mL} \cdot \mathrm{min}^{-1}$. Longer retention times and poor peak shape of Rasagiline mesylate was problem with the above method. Different columns such as YMC Pack ODS AM, Hypersil BDS and different buffers such as dipotassium hydrogen phosphate, Trifluoroacetic acid were also tried with different gradient methods to achieve the best 
chromatographic separation. But long retention times and poor peak shapes were still unavoidable. With $0.1 \%$ trifluoroacetic acid, Imp-A and Imp-B are co-eluting and long retention times are seen. Studied the separation and peak shape by varying $\mathrm{pH}$ from 2.5 to 10.0 with phosphate buffer, and observed that, as the $\mathrm{pH}$ is increasing towards 7.0, peaks were strongly retaining. Also at higher $\mathrm{pH}$, Rasagiline mesylate and Imp-A are coeluting. Added triethylamine to the mobile phase to study the separation on a $\mathrm{C} 18$ column at $6.5 \mathrm{pH}$ the peak shapes significantly improved but Rasagiline mesylate and Imp-A are still co-eluting. Changed the column to Agilent Zorbax SB-CN and obtained better separations and peak shapes with Solution A as 0.01 M Potassium phosphate and $1 \mathrm{~mL} \cdot \mathrm{Lt}^{-1}$ Triethylamine at $6.5 \mathrm{pH}$ and Solution B as Acetonitrile. But the oxidation degradant was not separating from the Rasagiline mesylate peak, making the method to modify further. The use of ion pair agent in acidic $\mathrm{pH}$ as buffer along with acetonitrile improved the peak shape of Rasagiline mesylate and obtained good resolution between all the impurities and Rasagiline mesylate. The \% of acetonitrile played a key role in the retention times and resolution between impurities. After many logical trials, chromatographic condition was established such that which could be suitable for separation of drug-degradation products and two known impurities. Using the optimized conditions, Rasagiline mesylate and its known impurities were well separated with a resolution of greater than 2 . In order to get better peak shape, monobasic potassium phosphate buffer used and carried the analysis. The Imp-A and Imp-B were clearly separated from the main peak with a resolution of 7.7 and 20.5, respectively, within a total run time of $4.17 \mathrm{~min}$ using gradient elution. The finalized chromatographic conditions are shown in section 2.3.

Conversion and Method optimization HPLC to UPLC: The main target of the chromatographic method was to achieve separation and quantification of rasagiline mesylate and its related impurities with a short run time. Initially, the gradient and isocratic HPLC conditions were optimized for rasagiline mesylate and its related impurities, which were then transferred to UPLC. A gradient system is always preferred over an isocratic system in order to achieve improved peak shape and resolution. Hence, it was decided to use a gradient HPLC mode. The chromatographic separation was achieved on a Sunfire C18, 250 x $4.6 \mathrm{~mm}, 5 \mathrm{~mm}$ column maintained at $25^{\circ} \mathrm{C}$. In gradient mode, using a mobile phase consisting of a buffer of $5 \mathrm{mM}$ potassium phosphate and acetonitrile, there was a good separation of rasagiline mesylate and its related impurities, while it was found to be eluting at a higher retention time. The response of rasagiline mesylate and its related impurities were found to be adequate at $210 \mathrm{~nm}$. The basic chromatographic conditions, such as the stationary phase, solvents, and UV detection employed in HPLC, were taken into account while developing the new UPLC method. The detection wavelengths, column temperature, and solvent used in HPLC were kept constant. The C18 stationary phase was chosen in order to have similar chemistry to that used in the HPLC. In UPLC, an Acquity BEH C18, (50x 2.1) $\mathrm{mm}, 1.7 \mu \mathrm{m}$ column was employed for the separation. The injection volume was scaled to $2 \mu \mathrm{L}$ from $10 \mu \mathrm{L}$, as used in the finalized chromatographic conditions are shown in section 2.3.

\subsection{Comparison of methods}

Comparison study of chromatographic performance comparative data on chromatographic performance of HPLC (gradient) and UPLC (gradient) has been obtained by injecting a solution of rasagiline mesylate and its related impurities. The performance parameters of both the systems are shown in Table 1. It is observed that the elution time of rasagiline mesylate in UPLC was reduced by 12 -fold to that of a gradient mode. The resolution and theoretical plates obtained for rasagiline mesylate in UPLC show comparatively better separation efficiency than HPLC. Theoretical plates obtained for rasagiline mesylate in gradient mode HPLC was obviously higher and it is lesser than UPLC. The standard chromatogram of related impurities obtained from UPLC condition is depicted in Figure 2.

Fig.2 Chromatogram of standard solution

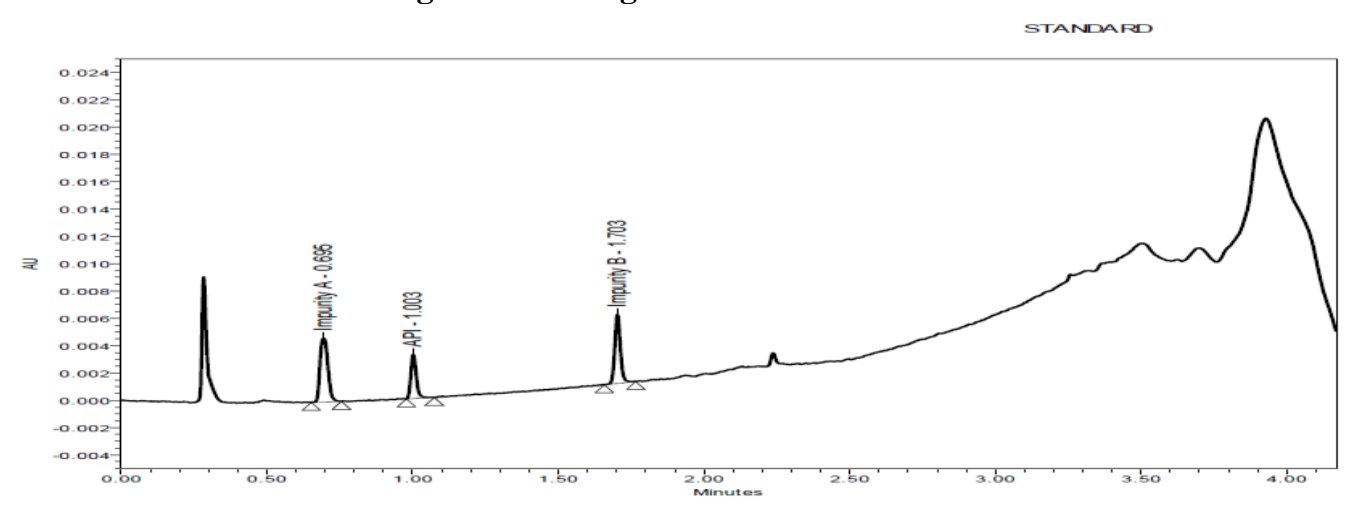


Development and Validation of a Stability-Indicating RP-UPLC Method for the Quantitative Analysis of antiparkinson drug and its related impurities

Table 1.A Comparison of System Performance of HPLC and UPLC for Rasagiline mesylate and its impurities

\begin{tabular}{|c|c|c|c|c|c|c|c|c|c|}
\hline Main features & \multicolumn{4}{|c|}{ HPLC } & \multicolumn{5}{|c|}{ UPLC } \\
\hline Flow $(\mathrm{mL} / \mathrm{min})$ & \multicolumn{4}{|c|}{1.0} & \multicolumn{5}{|c|}{0.5} \\
\hline \multirow{7}{*}{ Gradient program } & $\begin{array}{c}\text { Time } \\
\text { (minutes) }\end{array}$ & \multicolumn{2}{|c|}{$\begin{array}{l}\text { Buffer } \\
(\% \mathrm{v} / \mathrm{v})\end{array}$} & $\begin{array}{c}\text { Acetonitrile } \\
(\% \mathrm{v} / \mathrm{v})\end{array}$ & \multicolumn{2}{|c|}{$\begin{array}{c}\text { Time } \\
\text { (minutes) }\end{array}$} & \multicolumn{2}{|c|}{$\begin{array}{c}\text { Buffer } \\
(\% \text { v/v) }\end{array}$} & $\begin{array}{c}\text { Acetonitrile } \\
(\% \mathrm{v} / \mathrm{v})\end{array}$ \\
\hline & $\frac{0}{25}$ & \multicolumn{2}{|c|}{75} & 25 & \multicolumn{2}{|l|}{0} & \multicolumn{2}{|l|}{75} & 25 \\
\hline & 25 & \multicolumn{2}{|c|}{70} & 30 & \multicolumn{2}{|c|}{2.08} & \multicolumn{2}{|l|}{70} & 30 \\
\hline & 35 & \multicolumn{2}{|c|}{50} & 50 & \multicolumn{2}{|c|}{2.92} & \multicolumn{2}{|l|}{50} & 50 \\
\hline & \multirow{2}{*}{\multicolumn{2}{|c|}{\begin{tabular}{c|}
45 \\
45.1
\end{tabular}}} & 50 & 50 & \multicolumn{2}{|c|}{3.75} & \multicolumn{2}{|l|}{50} & 50 \\
\hline & & \multicolumn{2}{|c|}{75} & 25 & \multicolumn{2}{|c|}{3.77} & \multicolumn{2}{|l|}{75} & 25 \\
\hline & $\frac{45.1}{50}$ & 7 & & 25 & 4.17 & & 75 & & 25 \\
\hline Injection volume $(\mu \mathrm{L})$ & & & & & & & 2 & & \\
\hline Run time (minutes) & & & & & & & 4. & & \\
\hline USP Pocolution & & p-A & & p-B & & & $\overline{\mathrm{A}}$ & & Imp-B \\
\hline 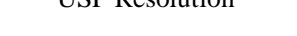 & & 1 & & 6.3 & & & 7 & & 20.5 \\
\hline USDT Tiling & & p-A & PI & np-B & & -A & $\mathbf{A}$ & & Imp-B \\
\hline USF laming & & .2 & .0 & 1.3 & & & 1 & & 1.0 \\
\hline USP Plate counts & & p-A & PI & np-B & & -A & $\mathbf{A}$ & & Imp-B \\
\hline USP FTate counts & & 983 & 205 & 20521 & & 93 & 32 & & 91693 \\
\hline & Inj.\# & Imp-A & API & Imp-B & Inj.\# & Imp & & API & Imp-B \\
\hline & 1 & 42862 & 21190 & 33610 & 1 & 892 & & 4072 & 6784 \\
\hline & 2 & 41538 & 21539 & 33650 & 2 & 891 & & 4146 & 6638 \\
\hline & 3 & 41692 & 21980 & 33143 & 3 & 892 & & 4043 & 6681 \\
\hline of RSD of Peak Area & 4 & 41240 & 21787 & 33864 & 4 & 886 & & 4027 & 6822 \\
\hline$\%$ KSD or Peak Area & 5 & 41014 & 21122 & 34882 & 5 & 886 & & 4083 & 6770 \\
\hline & 6 & 41521 & 21639 & 34058 & 6 & 876 & & 4034 & 6678 \\
\hline & Mean & 41644.5 & 21542.8 & 33867.8 & Mean & 8877 & & 4067.5 & 6728.8 \\
\hline & SD & 643.8 & 335.1 & 583.8 & SD & 60. & & 44.2 & 72.9 \\
\hline & \% RSD & 1.5 & 1.6 & 1.7 & $\%$ RSD & 0.7 & & 1.0 & 1.0 \\
\hline Cost per sample analysis & & & & & & & $\$ 2$ & & \\
\hline
\end{tabular}

\subsection{Analytical method validation}

\subsubsection{Linearity}

Solutions for assay linearity were prepared from API stock solution at 5 different concentration levels ranging from 40-160\% (i.e. 40, 80, 100, 120 and 160\%) of assay sample concentration. To demonstrate the linearity of detector response for API, Imp-A and Imp-B, injected the solutions of concentrations ranging from LOQ level to $200 \%$ of the target concentration of API and its impurities. Plotted a graph between peak area and concentration, and results are summarized in Table 2. Calculated the correlation co-efficient and found to be greater than 0.999 indicates that magnificent correlation between the analyte concentration and the peak area. Calculated the correlation co-efficient and found to be greater than 0.99 indicates that magnificent correlation between the analyte concentration and the peak area. The linearity graph for API, Imp-A and Imp-B were shown in Fig.3

Table 2.LOD, LOQ, linearity and precision data

\begin{tabular}{|c|c|c|c|}
\hline Parameter & API & Imp-A & Imp-B \\
\hline LOD (\%) & 0.006 & 0.003 & 0.006 \\
\hline $\mathrm{S} / \mathrm{N}$ ratio & 2.7 & 3.1 & 3.2 \\
\hline LOQ (\%) & 0.02 & 0.01 & 0.02 \\
\hline $\mathrm{S} / \mathrm{N}$ ratio & 9.8 & 10.2 & 10.6 \\
\hline \multicolumn{4}{|l|}{ Regression statistics } \\
\hline Slope & 40 & 86 & 66 \\
\hline Intercept & 98.4 & 338.3 & 155.7 \\
\hline Correlation coefficient & 0.9997 & 0.9999 & 0.9998 \\
\hline Method precision $(\% \text { RSD })^{\mathbf{a}}$ & 1.09 & 0.68 & 1.08 \\
\hline Intermediate precision (\% RSD) $^{\mathbf{a}}$ & 0.69 & 0.53 & 0.97 \\
\hline
\end{tabular}


Fig.3 Linearity graph for API, Imp-A and Imp-B

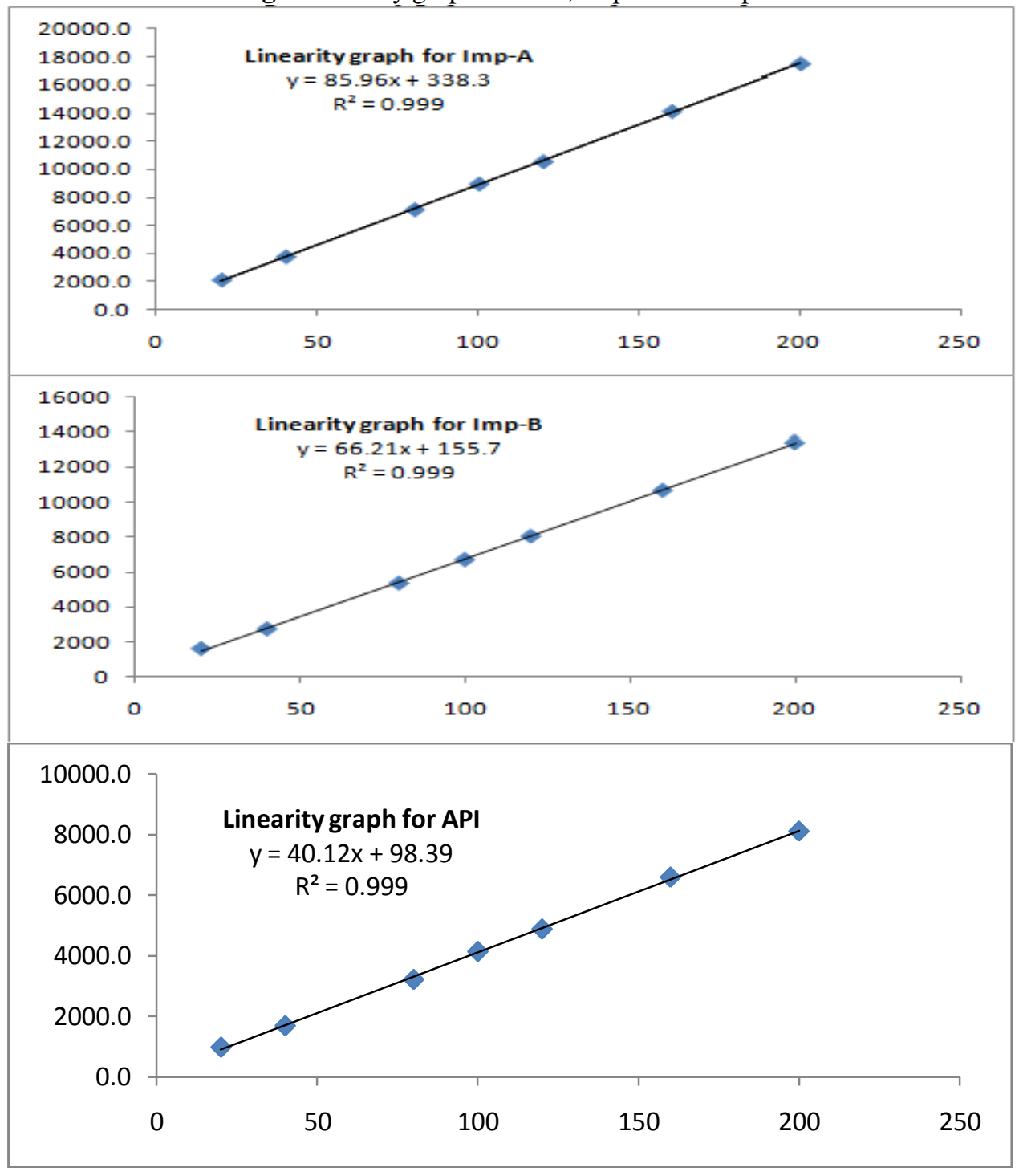

\subsubsection{Limit of detection (LOD) and limit of quantification (LOQ)}

The LOD and LOQ of API and its impurities were determined by using the signal to noise approach as defined in ICH guidelines [15]. The limit of quantitation refers to the lowest amount of an analyte in a sample that can be quantitatively determined with suitable precision and accuracy. There are different approaches to determine the LOQ and LOD. Typically the concentration level that generates a signal-to-noise $(\mathrm{S} / \mathrm{N})$ of 10 is regarded as the LOQ and the concentration level that generates an $\mathrm{S} / \mathrm{N}=3$ is regarded as the LOD. Practically, however, different compounds can possess different LOQ and LOD concentrations at the selected UV detection wavelength of a specific method. Depending on the type of detectors used, such as a photodiode array detector or a dual wavelength UV detector, the S/N ratios can be very different at the selected LOQ and LOD levels. The LOD and LOQ results of API and known impurities were presented in Table 2.

\subsubsection{Accuracy}

Recovery experiments were carried out to confirm the accuracy of the proposed method. The accuracy of the related impurities was evaluated at 3 different concentration levels of LOQ, 100 and $160 \%$ to the specification limits in triplicate. The samples were analyzed by the proposed method and the percentage recoveries for each and every impurity at each level and each replicate were calculated.

For assay, recovery experiments were carried out by preparing the solutions at 40,100 and $160 \%$ to the test concentration by taking the sample $2 \mathrm{mg}$ of API, $5 \mathrm{mg}$ of API and $8 \mathrm{mg}$ of API in different $50 \mathrm{~mL}$ flasks. These solutions were further diluted to obtain the concentration of 40,100 and $160 \mu \mathrm{g} \mathrm{mL}^{-1}$. The percentage recoveries were calculated. The recovery results for assay as well as impurities were expressed in terms of mean 
percentage RSD. The percentage recoveries obtained for API, Imp-A and Imp-B were within $2 \%$ and the results were presented in Table 3 . The recovery results indicate that the method is accurate and also found that there was no interference.

Table 3.Accuracy results

\begin{tabular}{|c|c|c|c|c|}
\hline \multirow{2}{*}{ Amount added } & \multicolumn{3}{|c|}{ \% Recovery range for triplicate injections } & \multirow{2}{*}{$\%$ RSD $^{\mathrm{c}}$} \\
\cline { 2 - 4 } & $\mathrm{API}^{\mathrm{a}}$ & $\mathrm{Imp}^{\mathrm{b}} \mathrm{A}^{\mathrm{b}}$ & Imp-B $^{\mathrm{b}}$ & 0.78 \\
\hline $\mathrm{LOQ}^{\mathrm{b} \&} 40 \%{ }^{\mathrm{a}}$ & $99.1-100.3$ & $98.3-100.2$ & $97.8-99.4$ & 1.26 \\
\hline $100 \%$ & $99.7-100.8$ & $98.6-99.4$ & $98.7-99.4$ & 1.88 \\
\hline $160 \%$ & $99.6-100.5$ & $99.1-99.6$ & $99.2-99.8$ & \\
\hline
\end{tabular}

a $\%$ Recovery obtained from assay; ${ }^{\mathrm{b}} \%$ Recovery obtained from related substances; ${ }^{\mathrm{c}}$ Mean of triplicate determination at each level.

\subsubsection{Method precision}

Method precision was evaluated by injecting the 6 different preparations of the system suitability solution and calculated the \% RSD for each impurity. Method precision for assay was performed by injecting six independent assay preparations of API sample against qualified working standard and calculated the \% RSD. The percentage RSD for the area of API, Imp-A and Imp-B, and assay results obtained from the intermediate precision study were within $1.5 \%$, confirms good precision of the method. The calculated percentage RSD values were presented in Table 2 .

\subsubsection{Controlled degradation}

Degradation studies were performed to demonstrate selectivity and stability-indicating capability of the proposed method. The sample was exposed to acid $\left(1 \mathrm{~N} \mathrm{HCl}, 60^{\circ} \mathrm{C}\right.$ for 7 Days), base $\left(1 \mathrm{~N} \mathrm{NaOH}, 60^{\circ} \mathrm{C}\right.$ for 7 Days), oxidation $\left(0.05 \% \mathrm{H}_{2} \mathrm{O}_{2}, 25^{\circ} \mathrm{C}\right.$ for 15 Days), thermal solid state $\left(100^{\circ} \mathrm{C}, 7\right.$ days $)$, thermal liquid state $\left(80^{\circ} \mathrm{C}, 24\right.$ hours $)$, humidity $\left(75 \% \mathrm{RH}, 40^{\circ} \mathrm{C}, 7\right.$ Days) and photolytic (1.2 million lux hours, $200 \mathrm{w} \cdot \mathrm{hr} / \mathrm{m}^{2}, 18$ days) degradation conditions. Samples were withdrawn at appropriate times and subjected to UPLC analysis after suitable dilution $\left(100 \mu \mathrm{g} \mathrm{mL}^{-1}\right)$ to evaluate the ability of the proposed method to separate API from its degradation products. Photodiode array detector was employed to check and ensure the homogeneity and purity of API peak in all the stressed sample solutions.

The degradation study revealed that API was sensitive to peroxide compared to other degradation conditions. Rasagiline mesylate (API) was degraded during oxidation $\left(0.05 \% \mathrm{H}_{2} \mathrm{O}_{2}\right.$, at $25^{\circ} \mathrm{C}$ for 15 Days) at around 5\% level and assay was found to be $95 \%$. The API and its impurities were treated with different degradation conditions including acid, base, peroxide, photolytic, humidity and thermal degradations. The degradation results were shown in Table 4.

Table 4.Forced degradation results of API

\begin{tabular}{|c|c|c|c|c|c|}
\hline Degradation conditions & \% Assay for API & \% Imp-A & \% Imp-B & $\begin{array}{c}\text { \% Major } \\
\text { degradation product }\end{array}$ & Mass balance \\
\hline $\begin{array}{c}\text { Acid treatment } \\
\left(1 \mathrm{~N} \mathrm{HCl}, 60^{\circ} \mathrm{C}, 7 \text { days }\right)\end{array}$ & 100.80 & 0.01 & 0.01 & 0.02 & 100.84 \\
\hline $\begin{array}{c}\mathrm{Base} \text { treatment } \\
\left(1 \mathrm{~N} \mathrm{NaOH}, 60^{\circ} \mathrm{C}, 7 \text { days }\right)\end{array}$ & 100.50 & 0.01 & 0.01 & 0.02 & 100.54 \\
\hline $\begin{array}{c}\mathrm{H}_{2} \mathrm{O}_{2} \text { treatment } \\
\left(0.05 \% \mathrm{H}_{2} \mathrm{O}_{2}, \mathrm{RT}, 15 \text { days }\right)\end{array}$ & 95.00 & 0.01 & 0.01 & 4.56 & 99.58 \\
\hline $\begin{array}{c}\text { Thermal (solid state) - } \\
100^{\circ} \mathrm{C}, 7 \text { days }\end{array}$ & 100.70 & 0.01 & 0.02 & 0.02 & 100.75 \\
\hline $\begin{array}{c}\text { Thermal (liquid state) - } \\
80^{\circ} \mathrm{C}, 24 \text { hrs }\end{array}$ & 99.90 & 0.01 & 0.02 & 0.02 & 99.95 \\
\hline $\begin{array}{c}\text { Humidity-75\% } \mathrm{RH}, 40{ }^{\circ} \mathrm{C}, \\
7 \text { days }\end{array}$ & 99.90 & 0.01 & 0.02 & 0.01 & 99.94 \\
\hline $\begin{array}{c}\text { Photolytic-1.2 million lux hours, } \\
200 \mathrm{Wh} / \mathrm{m}^{2}, 18 \text { days }\end{array}$ & 99.50 & 0.01 & 0.01 & 0.02 & 99.54 \\
\hline
\end{tabular}

The degradation of API in thermal, humidity and photolytic conditions was observed to be lower. Spectral purity of API and its impurities in the chromatogram of all the exposed samples are obtained from PDA and found to be spectrally pure, indicating that there was no co-elution of peak at the retention time of the respective known and unknown impurities. The max plot chromatogram of degradation sample was also checked to ensure that no degradation peak is missed due to use of wavelength of $210 \mathrm{~nm}$. 
For assay, the forced degradation study was performed by injecting the degradation samples at the assay concentration leveli.e. $10 \mu \mathrm{g} \mathrm{mL}{ }^{-1}$ and calculated the assay percentage against the reference standard. A summation of assay value and degradation impurity levels generally may be used to obtain mass balance for the test sample and to see how closely these add up to $100 \%$ of initial value with due consideration of the margin of analytical error. Mass balance was calculated for all the degradation samples by summing all the peaks found in the sample analysis (area of API + area of all the degradants formed in the degradation) and found to be in between 99.5 and $100.8 \%$ (Table 4). The assay of rasagiline (API) is unaffected in the presence of impurities and degradation products thus confirm the stability indicating of the developed UPLC method.

\subsubsection{Robustness}

The robustness as a measure of method capability to remain unaffected by small, but deliberate changes in chromatographic conditions was studied by testing influence of small changes in mobile phase composition ( $2 \%$ absolute change), column temperature $\left( \pm 5^{\circ} \mathrm{C}\right)$ and flow rate $\left( \pm 0.1 \mathrm{~mL} \mathrm{~min}^{-1}\right)$. In all robust conditions, the resolution between API, Imp-A and Imp-B has been found to be greater than 2.0 and the resolution between API and Imp-B was not less than 5.0. The peak shapes are found to be good for all impurities and API in all conditions, the assay variability was within $\pm 2.0 \%$ and the discrepancy from the estimation of API impurities was within $\pm 2.0 \%$. So, the method was found to be robust with respect to variability in all robust conditions.

\section{Conclusion}

The newly developed stability indicating RP-UPLC method for determination of rasagiline mesylate and its related impurities were found to be capable of giving faster retention times, while maintaining good resolution than that achieved with conventional HPLC. The method was completely validated, showing satisfactory data for all the parameters tested. This method exhibited an excellent performance in terms of sensitivity and speed. It is a stability-indicating method suitable for the rapid analysis of a rasagiline mesylate pharmaceutical drug substance and quantification of its related impurities.

\section{Acknowledgements}

The authors wish to thank the management of Department of chemistry, Adhiyamaan Engineering College, Hosur, Tamil Nadu, India and Zhangshu Pharma limited (China) for supporting this work.

\section{References}

[1] Plumb, R.; Perez, J.C.; Granger, J.; Beattie, I.; Joncour, K.; Wright, A. Ultra-performance liquid chromatography coupled to quadrupole-orthogonal time-of flight mass spectrometry. J. Rapid Commun. Mass Spectrom. 2004, 18, 2331-2337.

[2] Wren, S.A.C.; Tchlitcheff, P. Use of ultra-performance liquid chromatography in pharmaceutical development. J. Chromatogr. A 2006, 1119, 140 .

[3] Wren, S.A.C.; Tchlitcheff, P. UPLC/MS for the identification of b-blockers. J. Pharm. Biomed. Anal. 2006, 40, 571-580.

[4] Li, R.; Dong, L.; Huang, J. Ultra-performance liquid chromatographytandemmass spectrometry for the determination of epirubicin inhuman plasma. Anal. Chim. Acta 2005, 546, 167-173.

[5] Van Deemter, J.J.; Zuiderweg, E.J.; Klinkenberg, A. Longitudinal diffusion and resistance to mass transfer as causes of non ideality in chromatography. Chem. Eng. Sci. 1956, 5, 271-289.

[6] Oldfield, V., Keating, G. M., \& Perry, C. M. Rasagiline: a review of its use in the management of Parkinson's disease. Drugs. 2007, 67 (12), 1725-1747.

[7] Gallagher, D. A., \&Schrag, A. Impact of newer pharmacological treatments on quality of life in patients with Parkinson's disease. CNS Drugs. 2008, 22 (7), 563-586.

[8] Binda, C., Hubalek, F., \& Li M. Binding of Rasagiline-related Inhibitors to Human Monoamine Oxidases: A Kinetic and Crystallographic Analysis. Journal of medicinal chemistry. 2005, 48 (26), 8148-8154.

[9] Lecht, S., Haroutiunian,S., Hoffman, A., \&Lazarovici, P. Rasagiline - a novel MAO B inhibitor in Parkinson's disease therapy. TherClin Risk Manag. 2007,3 (3), 467-74.

[10] Jerkovich, A. D., LoBrutto, R., \&Vivilecchia, R. V., LC-GC North. Am. Suppl. 2005, $23,15$.

[11] Leandro, C. C., Hancock, P., Fussel, R. J., \&Keely, B. J. J. Chromatogr., A. 2006, 1103, 94.

[12] Jin, H., Praveen Kumar, A., Paik, D. H., Ha, K. C., Yoo, Y. J., \& Lee, Y. I. Microchem. J. 2010, 94, 139

[13] Gross, M. L., J. Am. Soc. Mass Spectrom. 1994, 5, 57.

[14] Lacorte, S., \& Fernandez-Alba, A. R. Mass Spectrom. Rev. 2006, 25, 866.

[15] ICH - Harmonised Tripartity Guideline; Validation of Analytical Procedures: Text and Methodology Q2(R1), IFPMA: Geneva, 2005.

[16] M. Fernandez, E. Barcia, and S. Negro, Journal of Pharmaceutical and Biomedical Analysis, vol. 49, no. 5, pp. 1185-1191, 2009.

[17] R. Narendrakumar, G. Nageswara Rao, and P. Y. Naidu, International Journal of Applied Biology and Pharmaceutical Technology, vol. 1, no. 2, pp. 247-259, 2010.

[18] M. Vijayalakshmi, J. V. L. N. Seshagiri Rao, and A. Lakshmana Rao, Rasayan Journal of Chemistry, vol. 3, no. 4, pp. 621-624, 2010.

[19] G. Devalarao, S. Kathirvel, and S. V. Satyanarayana, Journal of Pharmacy Research, vol. 4, no. 1, pp. 61-62, 2011.

[20] B. Rama and K. Preeti, International Journal of Pharmaceutical Sciences Review and Research, vol. 5, no. 1, pp. 5-7, 2010.

[21] J. Chen, X. Duan, P. Deng, H.Wang, and D. Zhong, Journal of Chromatography B, vol. 873, no. 2, pp. 203-208, 2008. 